VoL. 76 (2007) [219-225]

\title{
A NOTE ON THE THEOREM OF BATUROV
}

\author{
María MuÑoz
}

\begin{abstract}
D.P. Baturov proved in 'Subspaces of function spaces' Vestnik Moskov University Series $I$ (1987) that Lindelöf degree equals extent for subspaces of $C_{p}(X)$ when $X$ is a Lindelöf $\Sigma$-space. We prove that if the Lindelöf degree of the subspace is "big enough" the equality is true for a topological space $X$ not necessarily Lindelöf $\Sigma$.
\end{abstract}

\section{INTRODUCTION}

Baturov proved in [5] that Lindelöf degree equals extent for subspaces of $C_{p}(X)$ when $X$ is a Lindelöf $\Sigma$-space. This classical theorem answers a question of $\mathrm{A}$. V. Arkhangel'skĭ. Later, Buzyakova established in [8] a connection between the $D$-property and Baturov's theorem. A topological space $X$ is a $D$-space if, and only if, given a neighbourhood assignment $\{N(x): x \in X\}$, that is, $x \in \operatorname{Int} N(x)$ for each $x \in X$, there is a closed discrete subset $D$ of $X$ such that $X=\cup\{N(x): x \in D\}$, [10], see also [3, 4, 12, 6]. Buzyakova showed that $C_{p}(X)$ is hereditarily $D$ whenever $X$ a compact space, see [8]. Gruenhage describes the theorem of Buzyakova as an "explanation" of Baturov's result, whose proof is quite technical. Recently, Gruenhage has proved the same result for $X$ a Lindelöf $\Sigma$-space, [13].

The theorem of Baturov is established in terms of Lindelö $\Sigma$-spaces. The notion of Lindelöf $\Sigma$-space was introduced in [17] (in different terminology $K$-countably determined space, $[\mathbf{1 8}]$ ). We use the following characterisation of Lindelöf $\Sigma$-spaces (see [18, pp. 9596]).

Definition 1.1: A topological space $X$ is said to be Lindelöf $\Sigma$-space if it is the image of some subset of $\mathbb{N}^{\mathbb{N}}$ under an upper semicontinuous compact-valued map.

The class of Lindelöf $\Sigma$-spaces is the minimal class which contains all second countable spaces and all compact spaces and is closed with respect to finite products, closed subspaces and continuous images.

The goal of this paper is to give a different point of view of the theorem in the way that the restrictive hypothesis is not supported by the space $X$ or even by the subspace of $C_{p}(X)$. Our result is better in the sense that $X$ is not necessarily a Lindelöf $\Sigma$-space.

Received 8th January, 2007

Copyright Clearance Centre, Inc. Serial-fee code: $0004-9727 / 07$ SA2.00+0.00. 
To achieve our aim, we use the invariant cardinal function index of $K$-determination. This cardinal function is close to the ideas of Nagami [17] and the cardinal functions defined by Hödel [14]. It has turned out to be an useful tool to establish relationships between different cardinal functions of a topological space [9]. The index of $K$ determination, $\ell \Sigma(X)$ of a topological space $X$, measures how the space $X$ is determined by its compact subsets via upper semicontinuous compact valued maps defined on metric spaces.

Now we can enunciate the theorem of Baturov in these new terms. We shall prove that for a subspace $Y$ of $C_{p}(X)$, the Lindelöf number of $Y$ is equal to the extent of $Y$, if the Lindelö number of the subspace is greater than or equal to the index of $K$-determination of $X$.

\section{NOtATION AND RESUltS}

All our topological spaces $X, Y$ are assumed to be Tychonoff. Every Tychonoff space has a compactification. We denote by $b X$ a compactification of $X$. Our basic references are $[15,2,11,16]$. A cardinal number $m$ is the set of all ordinals which precede it. In particular, $m$ is a set of cardinality $\mathfrak{m} . \mathfrak{m}^{+}$is the smallest cardinal after m. A cardinal function $\phi$ is a function from the class of all topological spaces (or some precisely defined subclass) into the class of all infinite cardinals such that $\phi(X)=\phi(Y)$ whenever $X$ and $Y$ are homeomorphic. The requirement that cardinal functions take on only infinite cardinals as values simplifies statements of theorems. We assume that to all the cardinal functions we add the countable cardinal $\omega$. The weight of $X, w(X)$, is the minimal infinite cardinality of a base for the topology of $X$. The Lindelof degree of $X$, denoted $\ell(X)$, is defined as the smallest infinite cardinal $m$ such that every open cover of $X$ has a subcollection of cardinality $\leqslant \mathrm{m}$ which covers $X$. The density of $X, d(X)$, is the minimal cardinal of an everywhere dense set in $X$. The extent of $X e(X)$ is the supremum of the cardinalities of discrete closed sets in $X$. The hereditary density of $X$, $h d(X)$, is $\sup \{d(Y): Y \subset X\}$. The tightness of a point $x$ in a topological space $X$, $t(x, X)$, is the smallest infinite cardinal number $\mathfrak{m}$ with the property that if $x \in \bar{A}$, then there exists $A_{0} \subset A$ such that $\left|A_{0}\right| \leqslant \mathrm{m}$ and $x \in \overline{A_{0}}$. The tightness of a topological space $X, t(X)$ is the supremum of all numbers $\tau(x, X)$ for $x \in X$. It is obvious that for every subspace $Y$ of $X, t(Y) \leqslant t(X)$. We say that a topological property $\mathcal{P}$ is hereditary for a space $X$ if every subspace of $X$ has the property $\mathcal{P}$.

A multi-valued map $\phi: X \rightarrow 2^{Y}$ is said to be upper semicontinuous if it is compact valued and upper semicontinuous, that is, for every $x \in X$ the set $\phi(x)$ is compact, nonempty and for every open set $V \subset Y$ with $\phi(x) \subset V$ there is an open neighbourhood $U$ of $x$ such that $\phi(U) \subset V$. In terms of nets, if $\phi: X \rightarrow 2^{Y}$ is an upper semicontinuous map, $\left(x_{j}\right)_{j \in J}$ is a convergent net to $x$ in $X$ and $\left(y_{j}\right)_{j \in J}$ is a net in $Y$ such that $y_{j} \in \phi\left(x_{j}\right)$ for every $j \in J$, then we have that $\left(y_{j}\right)_{j \in J}$ has a cluster point $y$ which belongs to $\phi(x)$. 
More about upper semicontinuous maps can be found in [7].

Definition 2.1: Let $X$ be a topological space. The number of $K$-determination of $X \ell \Sigma(X)$, is the smallest infinite cardinal number $\mathfrak{m}$ for which there are a metric space $(M, d)$ of weight $m$ and an upper semicontinuous map $\phi: M \rightarrow 2^{X}$ such that $X=\bigcup_{t \in M} \phi(t)$.

When $\ell \Sigma(X)$ is countable we simply get Lindelöf $\Sigma$-spaces. The behavior of this invariant cardinal with respect to the usual operations and more properties can be found in [9]. We include in the proposition that follows some properties of $\ell \Sigma$ to make this paper self-contained. All of them can be found in [9].

Proposition 2.2. Let $X$ be a topological space. Then:

(i) $\ell(X) \leqslant \ell \Sigma(X)$.

(ii) Let $X_{i}$ be a topological space for $i=1, \ldots, r$, then

$$
\ell \Sigma\left(\prod_{i=1}^{r}\left(X_{i}\right)\right) \leqslant \max \left\{\ell \Sigma\left(X_{i}\right) \vdots i=1, \ldots, r\right\}
$$

(iii) $t\left(C_{p}(X)\right) \leqslant \ell \Sigma(X)$.

Proof: (i) Let $(M, d)$ be a metric space with $w(M) \leqslant \ell \Sigma(X)$ and $\phi: M \rightarrow 2^{X}$ an upper semicontinuous map such that $X=\cup\{\phi(t): t \in M\}$. Let $\mathcal{O}=\left\{O_{i}: i \in I\right\}$ be an open cover of $X$. The set $\phi(t)$ is compact for every $t \in T$, hence there exists a finite set of indices $i_{1}^{t}, \ldots i_{n_{t}}^{t}$ such that $\phi(t) \subset \cup\left\{O_{i_{j}^{t}}: j=1, \ldots, n_{t}\right\}$. Since $\phi$ is upper semicontinuous, for the open set $O(t):=\cup\left\{O_{i_{j}^{t}}: j=1, \ldots n_{t}\right\}$ there exist an open set $U(t)$ in $M$ such that $\phi(U(t)) \subset O(t)$. Now the family, $\mathcal{U}=\{U(t): t \in M\}$ is an open cover of $M$. Since $w(M)=\ell(M)$, there exists a subcover $\{U(t): t \in S\}$ of $M$ with $|S| \leqslant \ell \Sigma(X)$. Thus,

$$
X=\bigcup_{t \in S} \phi(U(t)) \subset \bigcup_{t \in \mathcal{S}} O(t)=\bigcup_{t \in S} \cup\left\{O_{i_{j}^{t}}: j=1, \ldots, n_{t}\right\}
$$

and the proof is finished.

(ii) There exist a metric space $M_{i}$ with $w\left(M_{i}\right) \leqslant \ell \Sigma\left(M_{i}\right)$ and an upper semicontinuous map $\phi_{i}: M_{i} \rightarrow 2^{X_{i}}$ such that $X_{i}=\bigcup_{t \in M_{i}} \phi_{i}(t)$ for each $i=1, \ldots, r$. The space $M:=\prod_{i=1}^{r} M_{i}$ is a metric space with $w(M) \leqslant \sup \left\{w\left(M_{i}\right): i=1, \ldots, r\right\}$ and the multivalued map $\phi:=\phi_{1} \times \ldots \times \phi_{r}$ defined on $M$ which takes values in $2_{i=1}^{\prod_{i}} x_{i}$ is an upper semicontinuous map such that $\prod_{i=1}^{r} X_{i}$ is covered by the union $\bigcup_{t \in M} \phi(t)$.

(iii) This is a consequence of a theorem of Arkhangel'skiu, [1] see ([2, Theorem II.1.1]) which affirms that if $\ell\left(X^{n}\right) \leqslant m$ for every $n \in \mathbb{N}$ then $t\left(C_{p}(X)\right) \leqslant \mathfrak{m}$, where $m$ is 
an infinite cardinal number. Now (iii) is obvious because using 2.2 and 2.2 we have that $\ell\left(X^{n}\right) \leqslant \ell \Sigma\left(X^{n}\right) \leqslant \ell \Sigma(X)$ for every $n \in \mathbb{N}$.

We recall that a continuous map $f: X \rightarrow Y$ is perfect if $f$ is a closed map and all fibres $f^{-1}(y)$ are compact subsets of $X$.

Proposition 2.3. Let $X$ be a topological space. Then, there exist a metrisable space $M$ with $w(M) \leqslant \ell \Sigma(X)$, a topological space $Y$ with $\ell \Sigma(Y) \leqslant \ell \Sigma(X)$ and continuous functions $f: Y \rightarrow X$ and $p: Y \rightarrow M$ such that $f$ is onto and $p$ is a perfect map.

Proof: Let $M$ be a metric space with $w(M) \leqslant \ell \Sigma(X)$ and $\phi: M \rightarrow 2^{X}$ an upper semicontinuous map. We define $Y:=\{(t, x): x \in \phi(t)\} \subset M \times b X$. Now the set $Y$ is closed in $M \times b X$. Let $\left(t_{j}, x_{j}\right)_{j \in J}$ be a convergent net to $(t, x)$ in $M \times b X$. We shall prove that $(t, x) \in Y$, that is $x \in \phi(t)$. Since $\left(t_{j}, x_{j}\right)_{j \in J}$ converges to $(t, x)$, this implies that $\left(t_{j}\right)_{j \in J}$ converges to $t$ in $M$ and $\left(x_{j}\right)_{j \in J}$ converges to $x$ in $b X$. For each open neighbourhood $U$ in $b X$ of $x$ there exists $j_{U}$ such that if $j \geqslant j_{U}$ then $x_{j} \in U$. Since $\phi$ is upper semicontinuous, the net $\left(x_{j}\right)_{j \in J}$ has a cluster point $x^{*}$ in $X$ such that $x^{*} \in \phi(t)$. If we assume that $x \neq x^{*}$ then there exist open sets $V$ and $V^{*}$ in $b X$ such that $x \in V$, $x^{*} \in V^{*}$ and $V \cap V^{*}=\emptyset$. But now there exist an open set $U^{*}$ in $X$ such that $x^{*} \in U^{*}$ and $U^{*} \subset V^{*} \cap X$, and $j \geqslant j_{V}$ such that $x_{j} \in U^{*} \subset V^{*}$, but also $x_{j} \in V$, which is a contradiction, so $x=x^{*}$, that is $x \in X$ and $x \in \phi(t)$.

On the other hand, we have that $\ell \Sigma(M \times b X) \leqslant \max \{\ell \Sigma(M), \ell \Sigma(b X)\}$, by 2.2 (ii). $\ell \Sigma(b X)$ is countable since $b X$ is a compact set and $M$ is a metrisable space. Hence $\ell \Sigma(M) \leqslant w(M) \leqslant \ell \Sigma(X)$. The index of $K$-determination does not increase under closure [9], hence $\ell \Sigma(Y) \leqslant \ell \Sigma(M \times b X) \leqslant \ell \Sigma(X)$.

The maps $f: Y \rightarrow X$ and $p: Y \rightarrow M$ given by $f(t, x):=x$ and $p(t, x):=t$ are continuous. The space $X$ is covered by the union $\cup\{f(t, x)=x,(t, x) \in Y\}$, hence $f$ is onto. On the other hand, $p$ is a perfect map. In fact, the set $p^{-1}(t)=\{t\} \times \phi(t)$ is compact and we have by Kuratowski's theorem [11, Theorem 3.1.16] that $\tilde{p}: M \times b X \rightarrow M$ given by $\widetilde{p}(t, x)=t$ is closed. Since $Y$ is closed, the map $p$ is closed too and the proof is over. $D$

Although in the proof of the theorem that follows we follow the general argument used in the proof of the theorem of Baturov, we include all the steps and changes because of the technical arguments used in it.

THEOREM 2.4. Let $X$ be a topological space and $T \subset C_{p}(X)$ be a subspace such that $\ell(T) \geqslant \ell \Sigma(X)$ then $\ell(T)=e(T)$.

Proof: The inequality $e(T) \leqslant \ell(T)$ is always true. To prove the other inequality, we may assume that $X$ is a topological space such that there exists a continuous perfect map $p$ defined on $X$ which takes values in a metrisable space $M$ with $w(M) \leqslant \ell \Sigma(X)$. In fact, by proposition 2.3 , there exist a metrisable space $M$ with $w(M) \leqslant \ell \Sigma(X)$, a topological space $Y$ with $\ell \Sigma(Y) \leqslant \ell \Sigma(X)$, and maps $f: Y \rightarrow X$ continuous onto and 
$p: Y \rightarrow M$ continuous and perfect. Since $X$ is a continuous image of $Y$, the map $\tilde{f}: C_{p}(X) \rightarrow C_{p}(Y)$ given by $\tilde{f}(h):=h \circ f$ for every $h \in C_{p}(X)$ is a homeomorphic embedding. Let $T \subset C_{p}(X)$ be a subspace such that $\ell(T) \geqslant \ell \Sigma(X)$, then $\ell(\tilde{f}(T))$ $\geqslant \ell \Sigma(Y)$. This inequality follows since $\ell(\tilde{f}(T))=\ell(T) \geqslant \ell \Sigma(X) \geqslant \ell \Sigma(Y)$.

To prove that $\ell(T) \leqslant e(T)$ we shall prove that for every infinite cardinal $m \geqslant \ell \Sigma(X)$, if $\ell(T)>\mathrm{m}$, then $e(T)>\mathfrak{m}$.

We suppose $\ell(T)>\mathfrak{m}$. There is an open cover $\gamma$ of $T$ in which there is no subcover of cardinality less or equal to $\mathrm{m}$. We can assume that the elements of the cover $\gamma$ have the form

$$
U\left(x_{i_{1}}, \ldots, x_{i_{n}} ; O_{k_{1}}, \ldots, O_{k_{n}}\right)=\left\{h \in C_{p}(X): h\left(x_{i_{j}}\right) \in O_{k_{j}}, j=1, \ldots, n\right\},
$$

where $x_{i_{j}}$ are points in $X$ and $O_{k_{j}} \in \mathcal{O}$ for $j=1, \ldots, n$, where $\mathcal{O}=\left\{O_{i}: i \in \mathbb{N}\right\}$ is a standard countable base of $\mathbb{R}$. For every $n \in \mathbb{N}$ we define the family $\mathcal{O}^{n}:=\left\{O_{i_{1}}\right.$ $\left.\times O_{i_{2}} \times \cdots \times O_{i_{\mathrm{n}}}: O_{i_{j}} \in \mathcal{O}, j=1, \ldots, n\right\}$. The cardinality of $\mathcal{O}^{n}$ is countable, so we can consider $\mathcal{O}^{n}=\left\{O_{n, m}: m \in \mathbb{N}\right\}$. We denote $U_{n, m}(x)=U\left(x_{i_{1}}, \ldots, x_{i_{n}} ; O_{k_{1}}, \ldots, O_{k_{n}}\right)$ where $x=\left(x_{i_{1}}, \ldots, x_{i_{n}}\right)$ and $O_{n, m}=O_{k_{1}} \times \cdots \times O_{k_{n}} \in \mathcal{O}^{n}$. We define $A_{n, m}$ $=\left\{x \in X^{n}: U_{n, m}(x) \in \gamma\right\}$. We consider the subset $\mathbb{P} \subset \mathbb{N}^{2}$ defined as $(n, m) \in \mathbb{P}$ if, and only if, $A_{n, m} \neq \emptyset$. The family $\gamma$ can be represented as $\gamma=\cup\left\{\gamma_{n, m}:(n, m) \in \mathbb{P}\right\}$, where $\gamma_{n, m}=\left\{U_{n, m}(x): x \in A_{n, m}\right\}$. For every map $h \in C_{p}(X)$ and $n \in \mathbb{N}$ we denote by $h^{n}$ the map from $X^{n}$ to $\mathbb{R}^{n}$ such that $h^{n}\left(x_{1}, \ldots, x_{n}\right)=\left(h\left(x_{1}\right), \ldots, h\left(x_{n}\right)\right)$ and by $p^{n}$ the map from $X^{n}$ to $M^{n}$ such that $p^{n}\left(x_{1}, \ldots, x_{n}\right)=\left(p\left(x_{1}\right), \ldots, p\left(x_{n}\right)\right)$. With this notation, we have that for each $h \in T$ there exist $(n, m) \in \mathbf{P}$ and $x \in A_{n, m}$ such that $h^{n}(x) \in O_{n, m}$.

The fact that there is no subfamily of $\gamma$ of cardinality less or equal to $m$ which covers $T$ can be written as if for every $(n, m) \in \mathbf{P}$ we choose $B_{n, m} \subset A_{n, m}$ and $\left|B_{n, m}\right| \leqslant \mathfrak{m}$, then there is $g \in T$ such that $g^{n}\left(B_{n, m}\right) \cap O_{n, m}=\emptyset$ for every $(n, m) \in \mathbb{P}$.

We construct by transfinite induction a set $F=\left\{h_{\alpha}: \alpha<\mathfrak{m}^{+}\right\} \subset T$ which is closed and discrete in $T$.

Choose $h_{0} \in T$ arbitrarily, and suppose that we have the functions $\left\{h_{\beta} \in T: \beta<\alpha\right\}$ where $\alpha<\mathfrak{m}^{+}$.

For each $n \in \mathbf{N}$ the space $\mathbb{R}^{n \cdot r} \times M^{n}$ is a metrisable space and $w\left(\mathbb{R}^{n \cdot r} \times M^{n}\right)$ $\leqslant w(M) \leqslant \ell \Sigma(X),\left[11\right.$, Theorem 2.3.13]. The hereditarily density degree of $\mathbb{R}^{n \cdot r} \times M^{n}$ coincides with the weight, see [15, Theorem 8.1], hence $h d\left(\mathbf{R}^{n \cdot r} \times M^{n}\right) \leqslant \ell \Sigma(X)$.

For each finite collection $\beta_{1}, \ldots, \beta_{r}<\alpha$ and $n \in \mathbb{N}$, we consider the map $h_{\beta_{1}, \ldots, \beta_{r}}^{n}$ $=h_{\beta_{1}}^{n} \Delta \ldots \Delta h_{\beta_{r}}^{n} \Delta p^{n}$ which is the diagonal product of the maps $h_{\beta_{1}}^{n}, \ldots, h_{\beta_{r}}^{n}$ and, $p^{n}$ defined on $X^{n}$ and taking values in $\mathbb{R}^{n \cdot r} \times M^{n}$. Now we have that $h_{\beta_{1}, \ldots, \beta_{r}}^{n}$ is a perfect map since $p$ is perfect, [11, Theorem 3.7.11].

For each $(n, m) \in \mathbb{P}$ we can choose in $A_{n, m}$ a set $S_{\beta_{1}, \ldots, \beta_{r}}^{n, m}$ with $\left|S_{\beta_{1}, \ldots, \beta_{r}}^{n, m}\right| \leqslant \ell \Sigma(X)$ such that $h_{\beta_{1}, \ldots, \beta_{r}}^{n}\left(S_{\beta_{1}, \ldots, \beta_{r}}^{n, m}\right)$ is dense in $h_{\beta_{1}, \ldots, \beta_{r}}^{n}\left(A_{n, m}\right)$. We define $B_{n, m}^{\alpha}=\cup\left\{S_{\beta_{1}, \ldots, \beta_{r}}^{n, m}\right.$ : $\left.\beta_{1}, \ldots, \beta_{r}<\alpha\right\}$. It is obvious that $\left|B_{n, m}^{\alpha}\right| \leqslant \max \{\mathfrak{m}, \ell \Sigma(X)\}=\mathfrak{m}$. We assume that we 
have $B_{n, m}^{\alpha}$ for every $(n, m) \in \mathbb{P}$ and hence we obtain that there exists $h_{\alpha} \in T$ such that $h_{\alpha}^{n}\left(B_{n, m}\right) \cap O_{n, m}=\emptyset$ for every $(n, m) \in \mathbb{P}$. The construction of the set $F=\left\{h_{\alpha}: \alpha<\mathrm{m}^{+}\right\}$ is finished.

We show that $F$ is discrete and closed in $T$. Assume the contrary. Then there is a point $g \in T$ which is a limit for $F$. For some $(n, m) \in \mathbb{P}$ and $x_{g} \in X^{n}$, we have $g \in U_{n, m}\left(x_{g}\right)$, that is $g^{n}\left(x_{g}\right) \in O_{n, m}$. By proposition 2.2 (iii), $t\left(C_{p}(X)\right) \leqslant \ell \Sigma(X)$, and hence $t(T) \leqslant \mathfrak{m}$. Let $\alpha_{0}$ be the smallest $\alpha^{\prime}<\mathfrak{m}^{+}$such that $g$ is a limit point of the set $\left\{h_{\alpha}: \alpha<\alpha^{\prime}\right\}$, and we put

$$
P=\left\{h_{\alpha} \in F: h_{\alpha}^{n}\left(x_{g}\right) \in O_{n, m}, \alpha<\alpha_{0}\right\} .
$$

The set $\left(g^{n}\right)^{-1}\left(O_{n, m}\right) \cap \bigcap\left\{\left(h^{n}\right)^{-1}\left(h^{n}\left(x_{g}\right)\right): h \in P\right\}$ is not empty because $x_{g}$ belongs to the intersection. We define the set $N=\cap\left\{\left(h^{n}\right)^{-1}\left(h^{n}\left(x_{g}\right)\right): h \in P\right\} \backslash\left(g^{n}\right)^{-1}\left(O_{n, m}\right)$.

We distinguish two situations. If $N$ is empty, we put $p^{n}\left(x_{g}\right)=t$. The map $p^{n}$ : $X^{n} \rightarrow M^{n}$ is perfect; hence $\left(p^{n}\right)^{-1}(t)$ is compact. Since the set $\left(g^{n}\right)^{-1}\left(O_{n, m}\right)$ is open, there is a finite set $\left\{h_{\beta_{1}}, \ldots, h_{\beta_{r}}\right\} \subset P$ such that

$$
\Phi=\cap_{i=1}^{r}\left(h_{\beta_{i}}^{n}\right)^{-1}\left(h_{\beta_{i}}^{n}\left(x_{g}\right)\right) \cap\left(p^{n}\right)^{-1}(t) \subset\left(g^{n}\right)^{-1}\left(O_{n, m}\right) .
$$

Now, $\Phi=\left(h_{\beta_{1}, \ldots, \beta_{r}}^{n}\right)^{-1}\left(h_{\beta_{1}}^{n}\left(x_{g}\right), \ldots, h_{\beta_{r}}^{n}\left(x_{g}\right), t\right)$. The map $h_{\beta_{1}, \ldots, \beta_{r}}^{n}$ is closed and the set $\left(g^{n}\right)^{-1}\left(O_{n, m}\right)$ is open such that $\Phi \subset\left(g^{n}\right)^{-1}\left(O_{n, m}\right)$. Since $A_{n, m} \cap \Phi \neq \emptyset$, there exists a point $y \in h_{\beta_{1}, \ldots, \beta_{r}}^{n}\left(S_{\beta_{1}, \ldots, \beta_{r}}^{n, m}\right)$ such that $\left(h_{\beta_{1}, \ldots, \beta_{r}}^{n}\right)^{-1}(y) \subset\left(g^{n}\right)^{-1}\left(O_{n, m}\right)$, [11, Theorem 1.4.12]. Thus, there is a point $x \in S_{\beta_{1}, \ldots, \beta_{r}}^{n, m} \cap\left(g^{n}\right)^{-1}\left(O_{n, m}\right)$. But then, by construction, the function $h_{\alpha}$, for $\alpha>\max \left\{\beta_{i}: i=1, \ldots, r\right\}$, does not belong to the neighbourhood $U_{n, m}(x)$ and $g \in U_{n, m}(x)$. We define $\alpha^{*}=\max \left\{\beta_{i}: i=1, \ldots, r\right\}$. Now, $\alpha^{*}<\alpha_{0}$, and $g$ is a limit point for the set $\left\{h_{\alpha}: \alpha<\alpha^{*}\right\}$. But $\alpha_{0}$ is the smallest cardinal with that property and we get a contradiction.

On the other hand, if $N$ is not empty, let $x^{\prime} \in N$. Then $g^{n}\left(x^{\prime}\right) \neq g^{n}\left(x_{g}\right)$, since $g^{n}\left(x_{g}\right) \in O_{n, m}$. But $h^{n}\left(x^{\prime}\right)=h^{n}\left(x_{g}\right)$ for every $h \in P$. This implies that $g$ does not belong to the closure of $P$, which is a contradiction. We have proved that $F$ is discrete and closed in $T$ and the proof is finished.

The theorem of Baturov follows as corollary of the main theorem.

Corollary 2.5. (Theorem of Baturov, [5]) Let $X$ be a Lindelöf $\Sigma$-space and $T \subset C_{p}(X)$ a subspace of $C_{p}(X)$, then $e(T)=\ell(T)$.

\section{REFERENCES}

[1] A.V. Arkhangel'skiü, 'On some topological spaces that arise in functional analysis', Russian Math. Surveys 31 (1976), 14-30.

[2] A.V. Arkhangel'skiĭ, Topological function spaces, Mathematics and its Applications (Soviet Series) 78 (Kluwer Academic Publishers Group, Dordrecht, 1989). Translated from the Russian by R.A.M.Hoksbergen. 
[3] A.V. Arkhangel'skir and R. Buzyakova, 'Addition theorems and D-spaces', Comment. Math. Univ. Car. 43 (2002), 653-663.

[4] A.V. Arkhangel'skiŭ, ' $D$-spaces and finite unions', Proc. Amer. Math. Soc. 132 (2004), 2163-2170.

[5] D.P. Baturov, 'Subspaces of function spaces', Vestnik Moskov. Univ. Ser. I Mat. Mekh. 4 (1987), 66-69.

[6] C.R. Borges and A.C. Wehrly, 'A study on D-spaces', Topology Proc. 16 (1991), 7-15.

[7] B. Cascales and L. Oncina, 'Compactoid filters and USCO maps', J. Math. Anal. Appl. 282 (2003), 826-845.

[8] R. Buzyakova, 'Hereditarily D-property of function spaces over compacta', Proc. Amer. Math. Soc. 132 (2004), 3433-3439.

[9] B. Cascales, M. Muñoz, and J. Orihuela, 'Index of $K$-determination of topological spaces', (preprint).

[10] E.K. van Douwen and W. Pfeffer, 'Some properties of the Sorgenfrey line and related spaces', Pacific J. Math. 81 (1979), 371-377.

[11] R. Engelking, General topology (PWN-Polish Scientific Publishers, Warsaw, 1977). Translated from the Polish by the author, Monografie Matematyczne, Tom 60. [Mathematical Monographs, Vol. 60].

[12] W. Fleissner and A. Stanley, 'D-spaces', Topology Appl. 114 (2001), 261-271.

[13] G. Gruenhage, 'A note on D-spaces', Topology Appl. 153 (2006), 2229-2240.

[14] R. Hödel, 'On a Theorem of Arkhangel'skiř concerning Lindelöf p-spaces', Canad. J. Math. 27 (1975), 459-468.

[15] R. Hödel, 'Cardinal Functions I', in Handbook of Set-Theoretic Topology (Elsevier Science Publishers, 1984), pp. 1-61.

[16] J.L. Kelley, General topology (Springer-Verlag, New York, 1975). Reprint of the 1955 edition [Van Nostrand, Toronto, Ont.], Graduate Texts in Mathematics 27.

[17] K. Nagami, ' $\Sigma$-spaces', Fund. Math. 65 (1969), 169-192.

[18] A. Rogers and E. Jayne, Analytic sets (Academic Press, London, 1980).

Departamento de Matemática Aplicada y Estadística

Universidad Politécnica de Cartagena

(Murcia)

Spain

e-mail: maria.mg@upct.es 